\title{
Feature Perception in Broadband Sonar Analysis - Using the Repertory Grid to Elicit Interface Designs to Support Human-Autonomy Teaming
}

\author{
F. Mccabe \\ School of Engineering, University of Birmingham \\ fxm493@bham.ac.uk
}

\begin{abstract}
Broadband sonar analysis is used to identify contacts of interest and develop a tactical picture when operating underwater. Sonar analysts must identify significant features from hydrophone recordings and use these to determine the type of contact presented and in which direction it is moving. This is performed with no external views available, leading to dynamic levels of information uncertainty. This work explores how sonar analysts process sonar data and how this differs from the analysis performed by experienced sound engineers. Subject Matter Experts described aural and visual features from hydrophone recordings using a repertory grid method. This was used to gain access to the 'frames' that the different experts were using, described in terms of constructs and concepts. The concepts are used to design a novel visual display, Visual Intelligent Narrative for Autonomous Systems (VINAS), which is designed to aid contact classification.
\end{abstract}

\section{CCS CONCEPTS}

- Human-Centred Computing; • Human-Computer Interaction; • Empirical Studies in HCI;

\section{KEYWORDS}

Trust, Human-Autonomy Teaming, Explainable AI, Defence

\section{ACM Reference Format:}

F. Mccabe and C. Baber. 2021. Feature Perception in Broadband Sonar Analysis - Using the Repertory Grid to Elicit Interface Designs to Support HumanAutonomy Teaming. In Proceedings of the 2021 International Conference on Multimodal Interaction (ICMI '21), October 18-22, 2021, Montréal, QC, Canada. ACM, New York, NY, USA, 7 pages. https://doi.org/10.1145/3462244.3479918

\section{INTRODUCTION}

Sonar analysis is a highly refined skill, requiring analysts to identify, classify and monitor objects in their environment through the detailed interpretation of hydrophone recordings and visual frequency analysis. In this study, we explore the cognitive constructs professional audio analysts use to make sense of hydrophone recordings. In the future, autonomous, Artificial Intelligence (AI) systems are likely to be used to support sonar analysis (UK Development Concepts And Doctrine Centre, (2018)). However, the

Permission to make digital or hard copies of part or all of this work for personal or classroom use is granted without fee provided that copies are not made or distributed for profit or commercial advantage and that copies bear this notice and the full citation on the first page. Copyrights for third-party components of this work must be honored

For all other uses, contact the owner/author(s).

ICMI '21, October 18-22, 2021, Montréal, QC, Canada

(C) 2021 Copyright held by the owner/author(s)

ACM ISBN 978-1-4503-8481-0/21/10

https://doi.org/10.1145/3462244.3479918

\author{
C. Baber \\ School of Computer Science, University of Birmingham \\ c.baber@bham.ac.uk
}

introduction of AI systems can cause changes in work and team structure, creating additional supervisory tasks, potentially reducing SA and attention (Parasuraman et al., (2000)) and requiring a change in skills and training (Bradshaw et al., (2013)). Appropriate trust in autonomy is needed to ensure the systems are used appropriately, requiring a user to have a good understanding of the processes, performance, and purpose behind a system (Lee et al., (2004); Chancey et al., (2017); de Visser et al., (2018); Schaefer et al., (2019)). Therefore, AI systems should be able to explain the reasoning behind their decision-making in a way that a human agent would be able to follow or understand - a "narrative" behind the decision-making process, allowing them to assess whether these solutions are viable and sensical. To be able to explain that decision-making, common ground must be established between the human and the autonomous agent, providing a space where they can share and present information that can be understood by both parties. Therefore, by understanding how information is used in the classification process, it is possible to establish how autonomous systems should present their output in order to maximise trust in the information provided and minimise information ambiguity and uncertainty. The aim is to understand decision-making strategies used for contact classification, and how this task is performed by Royal Navy Sonar Operators compared with participants with experience of sound analysis but not of submarines (i.e., sound engineers). This study focused on the development of a visual tool which would allow the prominent aural features used in autonomous classification to be highlighted to a user in an understandable way, and whether it would be possible to distinguish different contacts using this. The research hoped to answer the question, "how do expert audio analysts classify sounds?" It explores how individual perceptions of sounds differ depending on expertise, domain, and how these perceptions can be translated into designs for a decision support interface.

\section{PROBLEM SCOPE}

The task of vessel classification using broadband sonar analysis relies on Sonar Operators (SOs) analysing hydrophone recordings to identify distinguishing features, and defining a contact solution containing type, bearing, speed and range information. When operating below periscope depth, a submarine crew must rely on information gathered from multi-sensor systems in order to gain Situation Awareness (SA) and build an accurate picture of their operational environment; no external views or data transfer from onshore is available. Very little has changed in terms of user interface for broadband analysis since the 1980s, suggesting scope to 
improve or augment these displays in order to improve classification decisions. Errors in classification contribute to collisions and near misses between Royal Navy (RN) submarines and commercial vessels (Marine Accident Investigation Branch, (2020); McCabe et al., (2020); Marine Accident Investigation Branch, (2015)).

The aural markers used in classification are subtle and require iterative analysis and thorough investigation. The variations within the sound are not easily identifiable without experience, and even with experience they can be difficult to distinguish. Analysts must work in high-pressure environments under severe time constraints and the features they are listening for can be ambiguous, and easily masked by environmental noise.

The process of classifying contacts involves abductive reasoning, with analysts observing "effects" in their environment to establish a most likely "cause". Analysts build a mental model of what they are listening to, identify the characteristics of its engine, and then build on this understanding by inferring the speed the parts are moving at and the speed of the vessel. They then use the speed and engine characteristics to identify a specific classification. The Sonar Trainer highlights a crucial factor - analysts are trying to identify the absence of the normal, and the presence of the abnormal. The real decision-making and cognitive work occur not by ticking off a set of criteria to reach a classification, but by dynamically interpreting signals to infer the behaviour of a contact, and identifying when more information is needed or when there is a contradiction in what is expected and what is presented. This process can be understood in terms of the Recognition Primed Decision (RPD) model. SOs bring disparate, unrelated pieces of information together and construct a narrative around those pieces of information, and then test this against what they know and can observe to identify areas which are contradictory. They then seek more information to refine their narrative of the situation. The RPD Model has been applied in complex naval environments in other work (Kaempf et al., (1996)) and the process of sonar analysis appears to exemplify the same principles and cognitive processes.

\section{METHOD}

The repertory grid is an interview technique and cognitive analysis tool, used to help identify commonalities and themes in how individuals relate and classify different ideas. It was developed to support psychotherapy interviews in the 1950s by Kelly. It is a cognitive mapping technique that seeks to discover how interviews construct internal representations of their environment (Curtis et al., (2008)). It is proposed that this is a useful means of gaining access to the 'frames' used in sound analysis, particularly when these frames might rely on tacit knowledge (Polanyi, (1962)) and be difficult to put into words.

The repertory grid was chosen for this study for a number of reasons. Firstly, it works to minimize bias by relying on perceptive constructs provided by participants based on objective stimuli. It is a fairly quick and unobtrusive method, which does not require lengthy observation of tasks, which is impractical in such a sensitive defence domain.

Other human factors methods, such as paired comparison or cognitive walkthrough, rely on observing actors carrying out activities in their operational environment. Cognitive walkthroughs, for example, are designed to evaluate a specific chain of activities or usage of equipment or an interface for their learnability or workload (Mahatody et al., (2010)). At the present time, sonar analysis does not utilise any AI systems, which, when introduced, require new methods of working and interfacing.

This study focused on understanding the cognitive and perceptual work carried out by sonar analysts when deriving a classification from hydrophone recordings, which is why the repertory grid method was chosen; this gives us insight into an individual's perceptions of the stimuli, providing an understanding of how a human would classify these sounds mentally, instead of focusing on the usage of an interface which does not incorporate the systems proposed to be beneficial in this work.

The study was performed by a group of four sound engineers with an average of five years' experience in audio recording and production. These were compared with three SMEs who had different levels of experience of sonar analysis. These were an ex-RN Sonar Operator (SO), whose main responsibility during active service was to monitor and classify contacts using sonar analysis; an ex-RN Officer of the Watch (OOW), whose main responsibilities would have been to ensure the maritime platform navigates safely and avoids collisions; and a current RN Sonar Trainer (ST) with experience in training future sonar analysis, plus additional experience of Anti-Submarine Warfare aboard a surface ship. To improve understanding of the tasks, information communication, and processes involved in sonar contact classification, Critical Decision Method interviews were performed with two SMEs who had performed or used sonar analysis in their work, the SO and the OOW. Critical Decision Method interviews use cognitive probes to understand the decision-making processes utilized in high timepressure, high information-content, volatile environments (Klein et al., (1989)). These interviews lasted around two hours each and provided a strong foundation of understanding for what information is utilized and communicated during the task, how, and to whom.

A sample of five hydrophone recordings were used to form the "elements" in the repertory grid. These identified different size vessels, with other prominent features audible such as trawling fishing nets, "blade slap" and "shaft rub" (Table 1).

These recordings were combined into ten "triads", i.e., sets of three recordings. There were ten unique triads in total. For each triad, participants were asked to describe how two of the recordings were similar, and distinct from, the third, providing a word or phrase which would become a construct in the repertory grid (column one in Figure 1). Once all ten triads had been assessed, participants were asked to define an opposing word or phrase for each construct, becoming the contrast (column seven in Figure 1). Each recording was then rated against each construct / contrast pair, i.e., with a ' 1 ' indicating that the recording fit the construct and ' 0 ' for the contrast. This process was repeated with triads of spectrograms, a visual representation of an audio signal over time containing frequency and signal strength information. Filling in the grids for the recordings and the spectrograms and discussing the responses with the participant took around an hour in total for each participant.

Derivation of the cognitive concepts from the repertory grid was based on the methodology outlined by (Baber, (1996)), (Baber, 
Table 1: A table describing the five repertory grid elements and their key aural features

\begin{tabular}{ll}
\hline Element & Description \\
\hline 1 & Medium Merchant Vessel \\
2 & Small Merchant Vessel \\
3 & Large Merchant Vessel characterised by "blade slap", meaning it is light/empty of cargo: The vessel will be riding high in \\
& the water with the propeller not fully submerged \\
4 & Large Merchant Vessel characterised by "shaft rub": the sound of poorly machined propeller shafts or worn bearings \\
5 & Fishing vessel characterised by "trawl noise", the sound of trawling nets behind a fishing vessel; heard as clunking and \\
& tinkling sounds as the bobbins and chains contact the seabed \\
\hline
\end{tabular}

\begin{tabular}{|c|c|c|c|c|c|c|c|}
\hline \multirow[b]{2}{*}{ Construct } & \multicolumn{5}{|c|}{ Element Number } & \multirow[b]{2}{*}{ Contrast } & \multirow[b]{2}{*}{ Fla } \\
\hline & 1 & 2 & 3 & 4 & 5 & & \\
\hline Diesel & 1 & 1 & 0 & 1 & 1 & Quiet & 3 \\
\hline In-out & 1 & 1 & $\mathbf{0}$ & 0 & $\mathbf{0}$ & Consistent & 5 \\
\hline Blade flutter & 1 & 1 & 1 & 1 & 0 & Blade slap & 3 \\
\hline Whine & 1 & 0 & 0 & 1 & 0 & Hum & 3 \\
\hline Engine & 1 & 0 & 0 & 1 & 1 & Muffled & 2 \\
\hline Diesel Engine & 1 & 1 & 1 & 0 & 1 & Steam generator & 3 \\
\hline Flutter & $\mathbf{1}$ & 1 & 0 & 1 & 1 & Compressed cavitation & 3 \\
\hline Cavitation & 0 & 1 & 1 & 0 & 0 & Shaft & 3 \\
\hline In-out & 1 & 1 & 0 & 1 & 0 & Consistent & 4 \\
\hline Blade flutter & 1 & 1 & 1 & 1 & 0 & Blade slap & 3 \\
\hline \begin{tabular}{|l|} 
Total \\
\end{tabular} & 9 & 8 & 4 & 7 & 4 & & \\
\hline Template & 1 & 1 & 0 & $\mathbf{0}$ & 0 & & \\
\hline
\end{tabular}

Figure 1: An example of a completed repertory grid performed with the ex-SO SME with hydrophone elements

(2015)). Once the repertory grid table was constructed (Figure 1), analysis develops by creating a 'template' that splits responses into roughly two groups. This allows the constructs to be translated into a smaller number of hypothetical variables (Concepts) which explain the maximum possible variance. This was done by totalling the scores for each element and picking a value to split the scores, i.e., total row in Figure 1. In this example, two of the values are 8 or above and three are below 8 . All columns with a total below the chosen value are recorded as "0" and each with a total of the chosen value or over is recorded as a " 1 ". This template is then compared to each construct row. The total matches or "hits" for each construct is recorded, shown in column "Fla" (Figure 1). Constructs are grouped together as a Concept, removed from the grid, and the analysis is performed again until all constructs are conceptualised. From Figure 1, it can be seen that the construct pair "in/out, consistent" matches the template completely, and so becomes its own grouping. This is then removed from the grid and the analysis is repeated to find the next conceptual grouping.

These concepts were then compared and contrasted across roles and expertise in sonar analysis, eliciting information requirements. It was clear that the SO and OOW had very distinct information requirements to perform their roles. This allowed for the identification of which concepts were important to visualise in terms of classification. Concepts were visualised in a grid design and coloured depending on how many of the constructs within the concept had been recorded as fitting the signal (see Figure 2).

\section{RESULTS}

The way the sounds were interpreted differed depending on the level of domain knowledge and role of the participant. As we might expect, there was a difference in the interpretation of the sounds when comparing participants with previous experience of sonar analysis to those who had none. There were large differences between the constructs and concepts between Submariners and the sound engineers. Further, while the SO, OOW and ST had some similarities in their constructs, their overarching concepts differed, and the concepts show concentration dependant on their role, therefore having different supporting informational requirements.

\subsection{Hydrophone Recordings}

Focusing on the perception of audio recordings, there was a difference in response across all participants, in terms of focus and level of detail of analysis.

The sound engineers tended to interpret the recordings through a frame focused on recording quality. Their interpretations did not seek to identify semantics but focused on the aural properties of the sounds. Constructs identified using the repertory grid concerned clarity, pitch, and rhythm, which seems to match with their expected domain knowledge, involving the production of high quality, clear audio signals. This could be described as surface analysis the sound engineers were not interested in identifying what was being recorded, but instead, explored the recording medium itself. The construct "mechanical" was provided by $75 \%$ of participants, however no mechanism was identified, in contrast to the responses of participants familiar with sonar analysis, who all identified a mechanism ("engine", "diesel"), but to a different resolution depending 
Table 2: The conceptual groupings of constructs for SO listening to recordings

\begin{tabular}{lll}
\hline Concept & Construct & Contrast \\
Motion & In-out & Consistent \\
Signature & Diesel & Quiet \\
& Blade flutter & Blade slap \\
& Whine & Hum \\
Clarity & Flutter & Compressed Cavitation \\
& Engine & Muffled \\
Mechanism & Cavitation & Shaft \\
\hline
\end{tabular}

Table 3: - The conceptual groupings of constructs for OOW listening to recordings

\begin{tabular}{lll}
\hline Concept & Construct & Contrast \\
Range & Diesel engine close range & Gas turbine long range \\
Sound Source & Engine sounds & Biological sounds \\
& Machinery & Organic \\
& Drive train & Oars \\
& Close range & Long range \\
& Machinery noise & Human noise \\
Mechanism & Mechanical & Biological \\
Identify & Other internal systems & Propulsion chain \\
\hline
\end{tabular}

on their focus. Uniquely, the sound engineering group commented on frequency information within the recordings without reference to the spectrograms, inferring frequency information from audio analysis in contrast to visual analysis.

The SO and ST conceptualised the sounds into categories related to engine characteristics and movement. The SO inferred noises relating to engines in every recording, unlike the sound engineers, and at a finer resolution in comparison to the OOW. For the SO, the focus was on identifying characteristics of the engine, to identify engines, mechanisms and auditory features of engine propellers and shafts, grouped under the concept of "signature" (Table 2). By determining a specific combination of these sounds and configurations of propellers and shafts, it is possible to classify a contact, so this is aligned with the SO's main duties, which are to detect, track and classify contacts.

Interestingly, the SO uses "diesel" as a construct which contrasts with "quiet", but also "diesel engine", contrasted with "steam generator". The word "diesel" is used in two distinct ways; in the former, in the sense of safety (as without the presence of diesel noise, there is uncertainty of the contact's location), grouped in the concept of "signature", and in the latter pair, as an engine mechanism, grouped in the "mechanism" concept. Clarity was one important conceptualisation the SO made, encompassing the construct contrast pair "muffled" and "engine", as the clearer the recording, the better suited it is for analysis, so in this sense the clarity of the engine characteristics in the recording are important. This is a different conceptualisation of "clarity" when compared with the sound engineers, who were interested in the quality of the medium itself and not the quality of a specific signal within the medium.
The OOW is responsible for the overall safety of a vessel and can override navigational decisions to maintain safety of a vessel (Royal Navy, (2017)). This means they are interested in the physical location of a contact in relation to their vessel, trying to create a three-dimensional mental model of their environment, therefore prioritising range in their conceptualisation is aligned with their primary role. Like the $\mathrm{SO}$, the $\mathrm{OOW}$ was interested in the presence of mechanisms (Table 3), but to a less granular degree; instead of identifying propeller and shaft configurations, they were trying to identify the pattern of life, whether the sounds were mechanical or organic or contained human noise. Safety was their largest encompassing conceptualisation, involving the identification of human noise, internal mechanism noise and engine noise, using this and the range conceptualisation to help visualise the objects in their environment and their positional relationship to each other and themselves. Broadly they were interested in whether the noise was derived from an engine or a biological source, and how close it was. Again, this is aligned with their main responsibility of safety and collision avoidance. They need less detailed information about what was used in the classification, but more information pertaining to locality in order to effectively make navigational decisions.

\subsection{Spectrograms}

The spectrograms provided the participants with additional information to the aural stimuli. Sound engineers gathered information about the smoothness of the signal and whether the visual pattern repeated over time or in a particular frequency band. Again, sound engineers were not interested in inferring classification characteristics from the visual information and instead described the visual 
pattern presented to them. The SO gained insight on more dimensions when presented with visualised frequency information. Their predominant conceptualisations were concerned with specific engine configurations and speed. When presented with the ability to visualise distinct frequencies present in the recordings, instead of simply identifying the presence of engine parts, the SO was concerned with the specific number of these components. Being able to distinguish specific configurations of propellers and propeller shafts, supplemented with aural information, allows an SO to make an initial classification of a contact. The SO built on their mental model by beginning to interpret movement and speed, concerned with constructs such as "engine RPM", "engine firing rate" and "shaft RPM". This shows a fusion of aural and visual information in their classification process, utilising both to build a more detailed understanding of the structure of a contact, as both are required in order to identify the presence, and number of, specific engine components, and interpret their behaviour.

The OOW interpreted the visual information in a similar way to the sound engineers, conceptualising the shape and rhythm of the signal presented, and identifying patterns in its appearance. Like the SO, they infer additional movement information from the spectrograms, conceptualising whether there is rotation occurring.

The ST was concerned with identifying visual patterns in the spectrograms, focusing on the behaviour of the signal in terms of frequency intensity, diffusion and fundamentals. They conceptualised the "pattern" of the signal, its level of "regularity", its "similarity" and its "clarity"; here a methodological understanding can be seen, concerned with whether the signal has a consistent, recognisable pattern, or whether there are areas obscured by a lack of clarity, or dissimilarities. The ST spoke about the "absence of the normal, the presence of the abnormal", trying to find incongruencies between what is displayed and what they conceptualise as the classification, exampling a visual form of abductive reasoning.

Like the SO, the ST conceptualised "speed", with the pair "fast" and "slow", increasing understanding when presented with a visual representation of the signal, concerned with the movement of a contact.

\section{VINAS DESIGN}

Concepts elicited through the repertory grid technique were visualised in a grid. Concepts were coloured depending on how many "hits" for the constructs present within them were recorded for a given vessel recording.

Thus it was possible to see the differences in how each vessel had been conceptualised (Figure 2). This grid was derived from the SO repertory grid responses, but with the inclusion of "pattern" from the ST response as this concept contained distinct information that could be used in frequency analysis. This was compared with classification guidance, and we found a high level of agreement with processes employed in classification.

Figure 2 compares, from left to right, grids for: medium merchant vessel, small merchant vessel (top row), large merchant vessel with characteristic blade slap, large merchant vessel with characteristic shaft rub (middle row), fishing vessel trawling nets (bottom row), using a grid made for the role of SO, coloured using SO repertory grid responses. Concepts with all constructs present are coloured
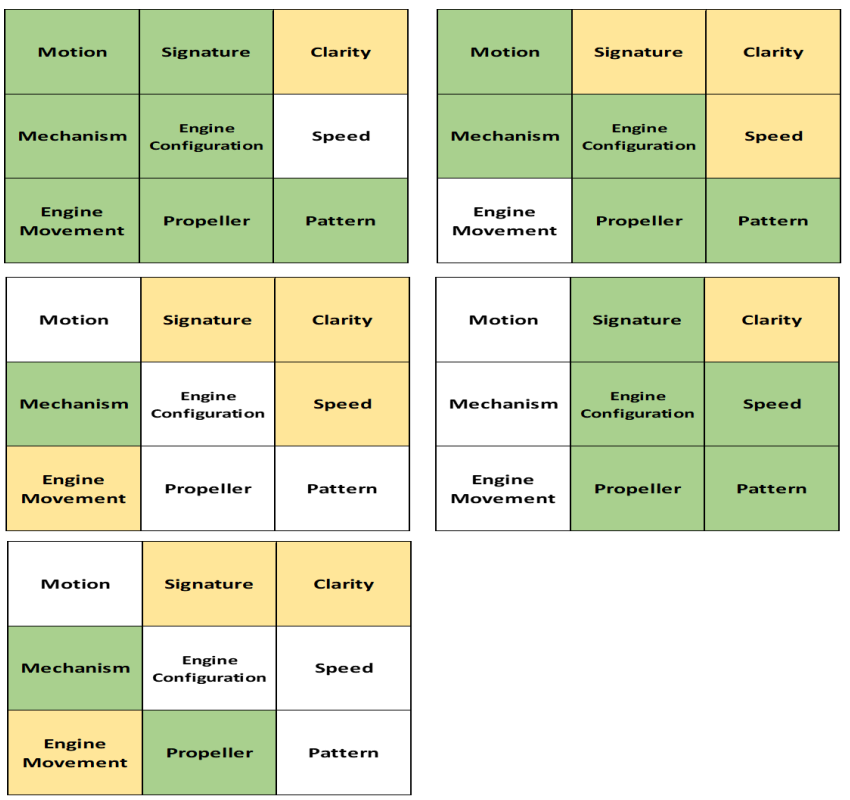

Figure 2: Completed VINAS Grids showing Distinguishing Colouration for Five Types of Vessel

green. Concepts with some constructs present are coloured yellow. Concepts with no constructs present are not coloured. As can be seen in Figure 2, each grid was distinct, depending on which vessel was being represented. Differences can be quickly visually assessed, and areas with few hits, showing a lack of information or ambiguity, can be quickly identified, and explored.

We propose that repertory grid provides a convenient means of identifying the frames used by sonar operators, and this, in the VINAS visualisation, provides an aide memoire that could be used to present the results from an autonomous decision aid. Central to this proposal is the suggestion that VINAS represents the narrative behind classification decisions. Coupled with confidence percentages for different classifications, this grid could highlight areas of ambiguity, abnormality or distinction to an operator quickly, allowing for speedy visual assessment of the autonomous agent's decision-making.

The proposed interface would include a spectrogram of the hydrophone recording for each contact, a method of playing a hydrophone recording, an autonomously generated suggestion for classification with a self-assessed confidence percentage, and the VINAS produced by the Machine Learning (ML) system from the sound. By displaying the VINAS alongside the suggested classification and confidence percentage assigned by the system, the user would have the ability to identify why a certain classification is presented, and potentially why a system is confident or unconfident in its proposed classification.

The idea behind an ML-generated VINAS would be to train a Deep Learning algorithm using the classifiers that were elicited through the repertory grid, so that the VINAS may be coloured depending on the number of classifiers found within the source signal. This would create common ground between the ML system and a 
sonar analyst so that their interpretations and classifications can be compared, with an explanation of the autonomously-generated decision provided by the VINAS grid, allowing for exploration of what markers were specifically identified within the source by the ML system.

ML has been used in other fields to generate classifications of sounds, such as in the speech recognition domain. However, the accuracy is lower when processing aural information when compared to visual information because of the dynamic and "transparent" nature of the frequencies within sound, when compared to the static and opaque values present within pictures (Wyse, (2017)). Accuracy improves when ML manages its own feature selection, and this is considered to be the preferred method when considering speech recognition (Wyse, (2017)), (Rothman, (2018)). Most high-accuracy speech recognition models utilise Hidden Markov Models instead of deep learning networks (Song, (2020)), however these require very clean samples, which is unsuitable for the problem at hand.

When considering hydrophone recordings, a much smaller set of classifiers would be used in training when compared with speech recognition, which means the accuracy may be improved in comparison.

In terms of the loss of accuracy when using pre-defined classifiers, although it may seem intuitive to always strive for greater accuracy from a system, when considering a safety-critical domain such as defence, comprehensive explanation is more desirable. This is because it is more important to be able to consistently identify when a system is incorrect, preventing dangerous and catastrophic consequences, than risk depending on an incorrect classification because it is not easy to discern that it is in error. In terms of trust, we hypothesise that trust would be better protected, even when a system is incorrect, when an explanation enables a user to consistently identify this, as predictability is important for trust development; it would be impossible to always predict when a system has made incorrect assumptions if no explanation is provided.

\section{DISCUSSION}

The interpretation of the sounds was directly influenced by the role of the SME. All conceptualised their interpretations of the sounds differently. This difference in depth of analysis and informational requirements must be reflected in what information is displayed to them.

Both the SO and the ST, for instance, identify specific engine features and rely on these to provide evidence to support initial classification. Therefore an autonomous agent needs a way to signify if these features were observed in its analysis as well as how certain it is that those specific features were observed. This would provide an SO with a comparison to what they observed, a way to quickly spot an absence of a classifier they would expect or the presence of an abnormality that affects their certainty in the classification, and a starting point for further investigation when there are inconsistencies or contradictions in the classifiers highlighted. The OOW does not require the same kind of display - they are interested in how close a contact is, whether it is dangerous, whether it is moving towards or away from them, whether it is a vessel or biological. For the OOW, information from sonar analysis is only one part of their tactical picture, and so the information they need is at a higher resolution. Command incorporates a larger wealth of information resources into their decision-making process when compared with the sonar team. Frequency characteristics are important for sonar analysis, but not so much for making navigational decisions. The SO requires that frequency information in order to develop their classification, but the OOW needs to know how close the contact is, directional information and speed, in order to assess any danger presented by the situation and develop a new course of action. However, a VINAS grid could be elicited from the OOW responses and still differentiated between each vessel.

Previous work has suggested that additional historic information about the operational area could be beneficial for Command, such as shipping timetables and historic AIS information (McCabe et al., (2020)). Although the information requirements are different, the interface designs must still be able to convey the information and the autonomous agent's certainty in that information in a way that makes the process, performance and purpose of the system evident, and highlights any areas of concern. Therefore all interfaces that incorporate an autonomous decision aid would be supported by the implementation of a VINAS, the contents of which would differ depending on the role and task. For the task of vessel classification, a VINAS highlighting which conceptual features were detected during autonomous analysis could be useful for an SO.

\section{CONCLUSIONS AND LIMITATIONS}

Although sample size was very limited, this work shows a visual interface concept designed to convey a contextual narrative behind an autonomous decision. Conceptual grids for an SO were presented, able to visually depict distinct patterns for five individual source signals, which is also true for the OOW responses. The VINAS design elicited from the SO conveyed the same classification markers outlined in a standard classification procedure. This shows potential for creating other grids that can convey an explainable story behind an autonomously generated classification, able to depict levels of uncertainty in a transparent way. Next stages for the research will be to test the efficacy of a VINAS grid and test how it affects user trust and confidence when dealing with high levels of information uncertainty.

\section{REFERENCES}

Baber, C. (1996). Repertory Grid and its Application to Product Evaluation. In Usability Evaluation in Industry (pp. 157-166).

Baber, C. (2015). Repertory Grid for Product Evaluation. In Handbook of Human Factors and Ergonomics Methods (pp. 31.1-31.7). London: Taylor and Francis.

Bradshaw, J. M., Hoffman, R. R., Woods, D. D., \& Johnson, M. (2013). The seven deadly myths of "autonomous systems." IEEE Intelligent Systems, 28(3), 54-61. https: //doi.org/10.1109/MIS.2013.70

Chancey, E. T., Bliss, J.P., Yamani, Y., \& Handley, H. A. H. (2017). Trust and the Compliance-Reliance Paradigm: The Effects of Risk, Error Bias, and Reliability on Trust and Dependence. Human Factors, 59(3), 333-345. https://doi.org/10.1177/ 0018720816682648

Curtis, A. M., Wells, T. M., Lowry, P. B., \& Higbee, T. (2008). An Overview and Tutorial of the Repertory Grid Technique in Information Systems Research. Communications of the Association for Information Systems, 23(November). https://doi.org/10.17705/ 1cais.02303

de Visser, E. J., Pak, R., \& Shaw, T.H. (2018). From 'automation' to 'autonomy': the importance of trust repair in human-machine interaction. Ergonomics, 61(10), 1409-1427. https://doi.org/10.1080/00140139.2018.1457725

Kaempf, G. L., Klein, G., Thordsen, M. L., \& Wolf, S. (1996). Decision making in complex naval command-and-control environments. Human Factors, 38(2), 220-231. https: //doi.org/10.1518/001872096779047986

Klein, G. A., Calderwood, R., \& Macgregor, D. (1989). Critical Decision Method for 
Eliciting Knowledge. Ieee Transactions On Systems Man And Cybernetics, 19(3), $462-472$.

Lee, J. D., \& See, K. A. (2004). Trust in Automation: Designing for Appropriate Reliance. Human Factors: The Journal of the Human Factors and Ergonomics Society, 46(1), 50-80. https://doi.org/10.1518/hfes.46.1.50_30392

Mahatody, T., Sagar, M., \& Kolski, C. (2010). State of the art on the cognitive walkthrough method, its variants and evolutions. International Journal of HumanComputer Interaction, 26(8), 741-785. https://doi.org/10.1080/10447311003781409

Marine Accident Investigation Branch. (2020). MAIB Report - Stena Superfast VII and Royal Navy Submarine. Southampton. Retrieved from www.gov.ul/maib

Marine Accident Investigation Branch. (2015). MAIBInvReport 20_2016 - Karen - Serious Marine Casualty. Retrieved from www.gov.uk/maib

McCabe, F., Baber, C., \& Stone, R. (2020). Classifying Vessels Using Broadband Sonar: Considerations for Future Autonomous Support. In R. Charles \& D. Golightly (Eds.), Contemporary Ergonomics \& Human Factors 2020 Conference Proceedings. Retrieved from https://publications.ergonomics.org.uk/uploads/Classifying-vesselsusing-broadband-sonar-considerations-for-future-autonomoussupport.pdf

Parasuraman, R., Sheridan, T. B., \& Wickens, C. D. (2000). A model for types and levels of human interaction with automation - Syst ems, Man and Cybernetics, Part A, IEEE Transactions on. IEEE Transactions on Systems, Man, and Cybernetics - Part A: Systems and Humans, 30(3), 286-297.

Polanyi, M. (1962). Tacit Knowing: Its Bearing on Some Problems of Philosophy. Reviews of Modern Physics, 34(4), 601-616.

Rothman, D. (2018). Human-Like Machine Hearing With AI (1/3) - Towards Data Science. Retrieved August 15, 2021, from https://towardsdatascience.com/humanlike-machine-hearing-with-ai-1-3-a5713af6e2f8

Royal Navy. (2017). BRD2: The Queens Regulations For the Royal Navy (pp. 1-11).

Schaefer, K., Baker, A., Brewer, R., Patton, D., Canady, J., \& Metcalfe, J. (2019). Assessing multi-agent human-autonomy teams: US

Song, Z. (2020). English speech recognition based on deep learning with multiple features. Computing, 102(3), 663-682. https://doi.org/10.1007/s00607-019-00753-0 UK Development Concepts And Doctrine Centre. (2018). Human-Machine teaming. Joint Concept Note 1/18 (Vol. 38).

Wyse, L. (2017). Audio spectrogram representations for processing with Convolutional Neural Networks, 1(1), 37-41. 